\title{
DISCLAIMER
}

This report was prepared as an wccount of work sponsored by an agency of the United States Government. Neither the United States Goyernment nor any agency thereol, nor any of their employess, makes any warranty, express or implied, or assumes any legal liability of responsibility for the accuracy, completeness, or usefulness of any information, apparatus, product. or process disclosed, or represents that its use would not infringe privately owned rights. Re[erence herein to any specific commercisl product, process, or service by trade name, trademark, manufacturer, or otherwise does not necessarily constitute or imply its endorsement, recommendation, or favoring by the United States Government or any agency thereof. The views and opinions of authors expressed herein do not necessarily state or reflect those of the Unied Stetcs Government of any agency thereof.

\section{ION THERMAL CONFINEMENT IN THE TFTR ENHANCED}

\section{CONFINEMENT REGIME}

R.J. Fonck, R. Howell ${ }^{+}$, K. Jaehnig, L. Roquemore, G. Schilling, S. Scont, M.C. Zarnstorff, M. Bitter, C. Bush, R. Goldston, H. Hsuan, D. Johnson, A. Ramsey, J. Schivell, and H. Towner Princeton Plasma Physics Laboratory, Princeton, NJ 08543, USA

\begin{abstract}
Measurements of the plasma ion temperature and toroidal rotation speed profiles have allowed the study of ion thermal transport in the TFTR hot ion enthanced confinement regime. Central ion temperatures up to $30 \mathrm{keV}$ and rotation speeds up to $8 \times 10^{5} \mathrm{~m} / \mathrm{sec}$ have been confirmed with new diagnostic measurements, and the ion thermal diffusivity is found to be non-neoclassical and comparable to the anomalous electron thermal diffusivity. The dominant effects of strong rotation are the down-shifting of the neutral beam energies in the plasma frame, which results in reduced ion and electron heating on axis, and the presence of off-axis ion heating from viscous damping of the plasma rotation.
\end{abstract}

+ Pennanent Address: Los Alamos National Laboratory, Los Alamos, New Mexico 
Since the discovery of the enhanced confinement energetic ion regime on the TFTR tokamak, ${ }^{l}$ considerable efforts have been made to perform detailed measurements of the ion energy confinement in order to improve our understanding of this regime. In actition to its intrinsic interest, an understanding of this regime is necessary in order to further pursue the goal of scientific breakeven for fusion power production in TFTR. To that end. we have performed a series of measurements of the radial profiles of the thermal ion temperature and ion toroidal rotation speeds. In addition to verifying the attainment of extremely high ion remperatures of $25-30 \mathrm{keV}$ in a tokamak plasma, these measurements have allowed us to examine the ion thermal transport in these plasmas and study the effects of plasma rotation on the plasma performance. The influence of plasma rotation on the plasma heating is a sopic of intense interest since the neutron production and energy confinement in TFTR plasmas have been found to depend strongly on the degree to which the neutral beam injection is balanced with respect to the torque applied to the plasma. 1,2

Central ion temperature measurements on early smaller tokarraks and the lack of good profile information led to a common assumption that the plasma ion thermal transport was reasonably described by a thermal diffusivity, $\boldsymbol{X}_{i}$, whose magnitude was typically up to a few times that predicted by neoclassical theory coupled with a convective power flux $=5 / 2$ $n_{i} T_{i}{ }_{1}$. As a consequence, ion thermal energy transport was not considered to be a dominant loss channel in neural-beam-heated plarmas. ${ }^{3-6}$ With the development of the charge-exchange recombination spectroscopy technique to provide reliable measurements of the ion temperature profile, that interpretation has come into question. The important results of Groebner et al. ${ }^{7}$ on the D-III tokamak indicated thar the $\chi_{i}$ in high density $H-$ mode plasmas was comparable to the electron thermal diffusivity $\chi_{e}$ in magnitude and radial dependence, and in disagreement with neoclassical predictions. Similar measurements on the PBX tokamak yielded less conclusive results because the neoclassical $\chi_{i}$ was comparable to both the measured values and the electron thermal diffusivity in the $r=a / 2$ region, and because electron-ion coupling and convective losses were dominant in 
the ion power balance. 8 Thus, it is interesting to study the ion thermal diffusivity and power $\mathrm{L}$ alance in the present generation of large, high-temperature tokamak experiments. The TFTR enhanced confinement regime offers an especially attractive environment for such ion power balance studies since the high electron temperatures and modest densities attained here result in almost negligible ion-electron coupling, and ion thermal conduction is the dominant ion energy loss mechanism over most of the plasma cross section.

The high-temperature enhanced confinement ("supershot") regime is attained in TFTR by neutral beam injection into a low recycling target plasma. The discharges studied here had a major radius of $2.45 \mathrm{~m}$ and a minor radius of $0.79 \mathrm{~m}$. Deuterium plasmas were heated by up to $20 \mathrm{MW}$ of deuterium neutral beam injection, with about $6 \mathrm{MW}$ counterangential to the plasma current, and the remaining power co-tangential. The highest values of stored energy, central ion temperature, and neutron smission were obtained with nearbalanced injection. With the availability of up to $=12 \mathrm{MW}$ of balanced beam power, maximum temperatures and neutron output were attained at discharge currents of 0.8 to 1.0 MA. The ion temperature profiles were highly peaked for the balanced injection cases, while they were broader for the lower confinement, purely co-injection cases with high toroidal rotation speeds, as was also the case for the measured electron density profiles.

A multispatial and multispectral imaging charge-exchange recombination spectroscopy diagnostic (CHERS) was installed on TFIR to provide radially resolved measurements of the ion temperature, $T_{1}(R, t)$, and toroidal rotation speed, $v_{\varphi}(R, t)$, for a wide range of discharge parameters. The fast doping neutrals needed for the charge-exchange excitation of visible lines from impurity ions were provided by either the heating neutral beams or a dedicated diagnostic neutral beam. UfF to 12 positions in the plasma could be measured simultaneously, with a $10-\mathrm{cm}$ spatial separation of the viewing sightlines for a single shot, but this could be reduced to $5 \mathrm{~cm}$ by changing the field of view between shots. The spatial resolution of the measurements was typically $3 \mathrm{~cm}$. For most of the measurements reported here, the CVI $5292 \AA$ ( $n=8-7$ ) transition was used, with estimated corrections 
applied to the measured line width and shift to account for the variation of the chargeexchange-tecombination rates with center-of-mass frame energy across the line profile. ${ }^{9}$ The central values of $T_{i}$ and $v_{\varphi}$ in the enhanced confinement regime measured by the CHERS diagnostic and by X-ray Doppler broadening measurements of the Ni XXVII Kalpha line are in agreement within their respective error bars.,2,10

The measured profiles have been analyzed with the SNAP (1D, equilibrium) and TRANSP ( $1-\mathrm{L} / 2 \mathrm{D}$, time-dependent) kinetic analysis codes, with an emphasis on deriving the ion thermal diffusivities and elucidating the effects of rotation on the themal ton power balance. These codes use, among other parameters, the measured profiles of ion and electron temperature, electron density, and plasma toroidal totation speed to derive the power balance for each plasma species. Both codes can be used in either an analysis mode, in which the measured $T_{i}(r)$ profiles are used as input to derive a value of $\chi_{i}(r)$ from the data, or in a predictive mode, in which a given model is chosen for $\chi_{i}(r)$ and the resulting calculated $\mathrm{T}_{\mathrm{i}}(\mathrm{s})$ profile is compared to the measurements. In these kinetic analyses, we assume classical electron-ion coupling and a spatially unifom $\mathbf{Z}_{\text {eff }}$ profile.

Comparisons of the measured $T_{i}(\tau)$ profiles with model transpor calculations from SNAP in the predictive mode show that the ion thermal diffusion is strongly nonneoclassical. For example, Fig- 1(a) shows the measured $T_{j}(r)$ profile for a 0.9-MA discharge with $13.6 \mathrm{MW}$ of balanced neutral injection, $\overline{\mathrm{n}}_{e}=2.5 \times 10^{19} \mathrm{~m}^{-3}$, and $\mathrm{n}_{e}(0)=$ $5.0 \times 10^{19} \mathrm{~m}^{-3}$. The solid points in the plot indicate measurements on the outboand side of the plasma center where the bearn-neutral density is higher and where the CHERS signals are most reliable. The open symbols indicate data from points inboard of the plasma center. The solid lines indicate the smoothed and fitted curves for $T_{i}(r)$ and $T_{e}(r)$ used for the transpor calculations in the analysis mode. Also shown in Fig. 1(a) are comparisons of the measured $T_{i} \Leftrightarrow$ profile with the values calculated from the SNAP code under the assumprions that $\chi_{i}=3 \times \chi_{i} \mathrm{NC}$, where $\chi_{i} \mathrm{NC}$ is the Chang-Hinton neoclassical ion thermal diffusivity, ${ }^{11}$ and $\chi_{i}(r)=2 \times \chi_{e}(r)$, where $\chi_{e}(r)$ is the electron thermal diffusivity derived from the measured $T_{e}(r)$ profiles. The measured $T_{i}(r)$ profile is clearly much narrower than 
that expected from neoclassical theory, while $\chi_{i}=2 \times \chi_{e}$ produces a much closer approximation to the measured values. Indeed, a survey of several discharges using the SNAP code in the analysis mode to derive a $\chi_{i}(R)$ profile indicates that $\chi_{i}=(1-3) \chi_{e}$ at $r / a$ $=0.5-0.7$, with similar radial profiles except in the innermost central region. More detailed modelling with the TRANSP code, which included the additional effects of beam-ion energy diffusion and beam-neutral charge exchange with unthermalized beam ions, indicates that $\chi_{i} \approx \chi_{e}$ is often a reasonable approximation for most of the glasma cross section.

While ion thermal convection is usually negligible over most of the minor radius, it is significant in the central plasma region $(\mathrm{r} / \mathrm{a}<0.2)$. Depending on the discharge conditions, a convective ion power flow given by $\mathrm{Q}_{\mathrm{i}}$, sonv $=(1.5) \mathrm{T}_{\mathrm{i}} \Gamma_{\mathrm{i}}$ (where $\Gamma_{\mathrm{i}}$ is the ion particle flux) is required to give good agreement with the measured profiles and to avoid unphysical negative $\chi_{i}$ s in the central plasma region. The deviation of the convective multiplier from the often-used $5 / 2$ value presumably reflects the nature of the assumed turbulence underlying this convective transport. ${ }^{12}$ For consistency, we use a value of $3 / 2$ in the analyses presented here.

High toroidal rotation speeds have a direct influence on the plasma performance in this hot ion regime, and measurements of the $T_{i}$ and $v_{\varphi}$ radial profiles help to quantify the effects of rotation on the plasma behavior. The overall neutron yield is lowered by the reduced relative energy between the fast ions and the rotating bulk plasma ions, and rotation has a strong effect on the plasma power deposition. ${ }^{13}$ The reduced relative speeds between the plasma and neutral beam particles result in reduced beam energy deposited in the plasma in its rotaing frame, and reduced beam penerration to the plasma core. This, in tum, iesults in a power deposition profile with less central ion and electron heating than in the non-rotating case. The viscous damping of the plasma rotacion is assumed to provide an additional source of heating power to the ions which, since it is strongest where the $v_{\varphi}(r)$ gradient is largest, peaks off axis. 
These combined effects of rotation lead to the broader $T_{i}(r)$ profiles observed in unbalanced injection cases, and, in extreme cases, have resulted in flat or hollow $T_{i}(r)$ profiles. For example, Fig. l(b) shows the measured and calculated ion temperature profiles for an unbalanced injection case. Here full co-injection of $11 \mathrm{MW}$ gives rise 10 a peaked $v_{\varphi}(r)$ profile (i.e., Gaussian in shepe with a half-width-at-half-maximum $=0.34 \mathrm{~m}$ ) with $v_{\varphi}(0)=8 \times 10^{5} \mathrm{~m} / \mathrm{sec}$ for a standard 0.9-MA discharge of $\tilde{n}_{e}=1.7 \times 10^{19} \mathrm{~m}^{-3}$ and $\Omega_{e}(0)=3.1 \times 10^{19} \mathrm{~m}^{-3}$. As with the balanced injection case, the ion thermal diffusivity $\chi_{i}$ is strongly non-neoclassical, and $\chi_{i}-\chi_{e}$ plus the effects of ion viscous heating reasonably reproduces the measured $T_{1}(r)$ profile. The analysis of the momentum transport in these plasmas will be reponed in a separate paper. 14

Figures 2(a) through (d) show the calculated heating power density profiles and the integrated power balance profiles for both the balanced injection and the fully co-injected discharges discussed above. To ease comparison between these different cases, we plot here the power densities divided by the volume-averaged total input heating power from the neutral beams (i.e., the absorbed beam power minus direct charge-exchange ion losses). Likewise, the ion power balance curves from SNAP shown in Figs. 2(c) and (d) give the volume-integrated power losses nonnalized to the total heating power. In the balanced injection case, the high relative beam energies lead to substantial direct ion and electron heating in the plasma center and strong central absorption of the neutral beams in these low recycling, low edge density discharges. In contrast, the down-shifted relative beam energies in the rotaning plasma case lead to reduced central heating of the ions and electrons and a somewhat broadened beam deposition profile. In addition, there is substantial offaxis heating of the ions due to viscous rotation damping.

The ion power balance for the balanced injection case confirms that the ion power losses are strongly dominated by thermal conduction (given by $n_{i} \chi_{i} \nabla T_{j}$ ) over most of the plasma cross section, except for the central r/a $\leq 0.2$ region, where conductive and convective losses are of equal magnitude. In some contrast, the ion power balance analysis 
of the rotating plasma [Fig. 4(d)] indicates that convection dominates the core power flow for $\mathrm{r} / \mathrm{a} \leq 0.3$, and it remains non-negligible (along with electron-ion coupling) beyond $\mathrm{r} / \mathrm{a}=$ 0.6 . The volume-integrated power inpur to the ions from viscous damping of the plasma rotation becomes a significant fraction of the overall input power to the ions for $\mathrm{r}>0.4 \mathrm{~m}$. In both cases, the consequence of $\left.\chi_{i}(r)=\chi_{j}, r\right)$ is that the ion loss channels are much more important in the overall power balances than for the case of neoclassical transport.

Given the strong role of balanced injection in the production of high stored energies and neutron Tuxes, it is of interest to ask whether the above-mentioned effects of rotation on the beam deposition are sufficient to produce the observed differences in overall confinement between balanced and unbalanced injection cases. To that end. Fig. 3 shows the comparisons of the inferred $\chi_{1}(r)$ and $\chi_{e}(r)$ for four balanced $\left(\tau_{E}=0.14 \mathrm{sec}\right)$ and wo unbalanced $\left(\tau_{E}=0.10 \mathrm{sec}\right)$ injection cases. These shots had $0.9 \mathrm{MA}$ with $\mathrm{P}_{[N]}=11 \mathrm{MW}$ for the co-injection cases, and 12-14 MW for the balanced cases. The rusults are plotted in Fig. 3 as a range of values of $\chi$ at each radius, with the range indicating the spread of values obtained from uie similur discharges. The uncertainties in the values of $\chi_{i}(r)$ and $\chi_{e}(r)$ from a single discharge, estimared fmm the uncertainties in the $T_{i}(r), T_{e}(r), Z_{e f f}$, and other parameters from a single shot, are 1 to 2 times larger than the ranges indicated in Fig. 3 for $r / a \geq 0.5$. At small radii, near the plasma center, large uncertainties in $\chi_{i}$ ard $\chi_{e}$ arise from uncertainties in the respective convective multipliers. As seen in Fig. $3, \chi_{i}(r)$ has a magnitude and radial dependence conparable to that of $\chi_{\mathrm{e}}(\tau)$, which agrees with the results from the $T_{i}$ simularions shown in Fig. 1. For comparison, the range of the values of $\chi_{i}{ }^{N C}$ derived from neoclassical theory is also shuwn in Fig. 3(a), and is seen to deviate strong!y from the range of values deduced from the kinetic measurements.

The thermal diffusivities themselves are not significantly different between cases of balanced and unbalanced injection. The observed differences in the ion temperature profiles are adequately explained by taking into account the down-shift of beam energies in the rotating plasma frame, a mild broadening of the beam deposition prufile, and tie occurrence of off-axis ion heating due to viscous rotational damping. 
In summary, new measurements of the ion temperature profile in TFTR indicate that the Son thenmal transpor is found to be very non-neoclassical in these large high-temperature plasmas. The anomalcus ion themal diffisivity is similar in magnitude and profile to the anomaicus electron thermal diffusivity, Analysis of the transport near the plasna axis in:ticates that the convective power flow is lower than used in earlier analyses of smaller experiments. The presence of strong toroidal rotation is manifested mainly by broadened temperature profiles due to down-shifting of the beam ion speeds in the plasma frame and the presence of off-axis heating due to viscous rotational damping.

The authors wish to thank K. Young, R. Hawryluk, D. Meade, the TFTR operations staff, and the TFTR technical crew for support during this work. Useful discussions with S. Kaye are alsø gratefully acknowledged. This work was supported by U.S. DoE Contract No. DE-AC02-76-CH0-3073. 


\section{References}

1 J. D. Strachan et al., Phys. Rev. Let. 58,1004 (1987).

2 M. Bitter et al., Plasma Physics and Controlled Fusion 29, 1235 (1987).

3 H. Eubank et al., Phys, Rev, Lett. 43, 270 (1979).

4 G. Becker et. al., Nucl. Fusion 23, 1293 (1983).

5 D. W. Swain et al., Nucl. Fusion 23, 1293 (1983).

6 S. M. Kaye et al., Nucl. Fusion 24, 1303 (1984).

7 R. J. Groebner et al., Nucl. Fusion 26, 543 (1986).

8 M. Okabayashi et al., Proceedings of the 11 th Intemational Conference on Plasma Physics and Controlled Nuclear Fusion Research, 13-20 Nov., 1986 Kyoto, Japan (IAEA, Vienna, 1987), 275.

9 R. B. Howell, R. Fonck, K. Jaehnig, and R. Knize, Princeton Plasma Physics Laboratory Report PPPL-2539, Aug., 1988; Rev. Sci. Instrum. (to be published).

10 R. J. Fonck et al. Proceedings of 15 th European Conference on Concolled Fusion and Plasma Heating, May 16-20, 1988, Dubrovnik, Ed. S. Pesic and J. Jacquinot (European Physical Society, 1988), I-83.

11 C. J. Chang and F. L. Hinton, Phys. Flutds 25, 1493 (1982).

12 M. C. Zamstorff et al., Proceedings of 15th European Conference on Controlled Fusion and Plasma Heating, May 16-20, 1988, Dubrovnik, Ed. S. Pesic and J. Jacquinot (European Physical Sociery, 1988), 1-95; Plasma Physics and Contolled Fusion (to be published).

13 R.J. Goldston, in Basic Physical_Processes of Toroidal Eusion Plasmas, ediced by G.P. Lampis et al. (Commission of the European Communities, Brussels. 1986), Vol. J, p. 165.

14 S. Scots et al., Proceedings of 15th European Conference on Controlled Fusion and Plasma Heating, May 16-20, 1988, Dubrovnik, Ed. S. Pesic and J. Jacquinor (European Physical Society, 1988), I-103. 


\section{Eigure Captions}

Figure 1. (a) Measured and simulated ion temperature profiles for a balanced injection discharge. Closed symbols: measurements for $\mathrm{R} \geq \mathrm{R}$ pl; open symbols: $\mathrm{R}$ $<\mathrm{R}_{\mathrm{pl}}$, where $\mathrm{R}_{\mathrm{pl}}$ is the major radius of the plasma axis. The smoothed $T_{e}$ profile is also shown. (b) Same as (a) but for sull cc-injection with $v_{\varphi}(0)=8$ $\times 10^{5} \mathrm{~m} / \mathrm{sec}$.

Figure 2. Comparison of input power densities and volume-integrated ion power balance for a nunrotating and strongly rotating discharge. For the balanced case (a,c), $P_{\text {inj }}=13.6 \mathrm{MW}$ and $P_{\text {absorbed }}=12.8 \mathrm{MW}$, while $P_{\text {inj }}=11.0 \mathrm{MW}$ and $P_{\text {absorbed }}=10.4 \mathrm{MW}$ for the co-injection case $(b, d)$.

Figure 3. Ion (a) and electron (b) thermal diffusivities for nonroiating and rotating discharges. The neoclassical ion diffusivity range includes both types of discharges. 

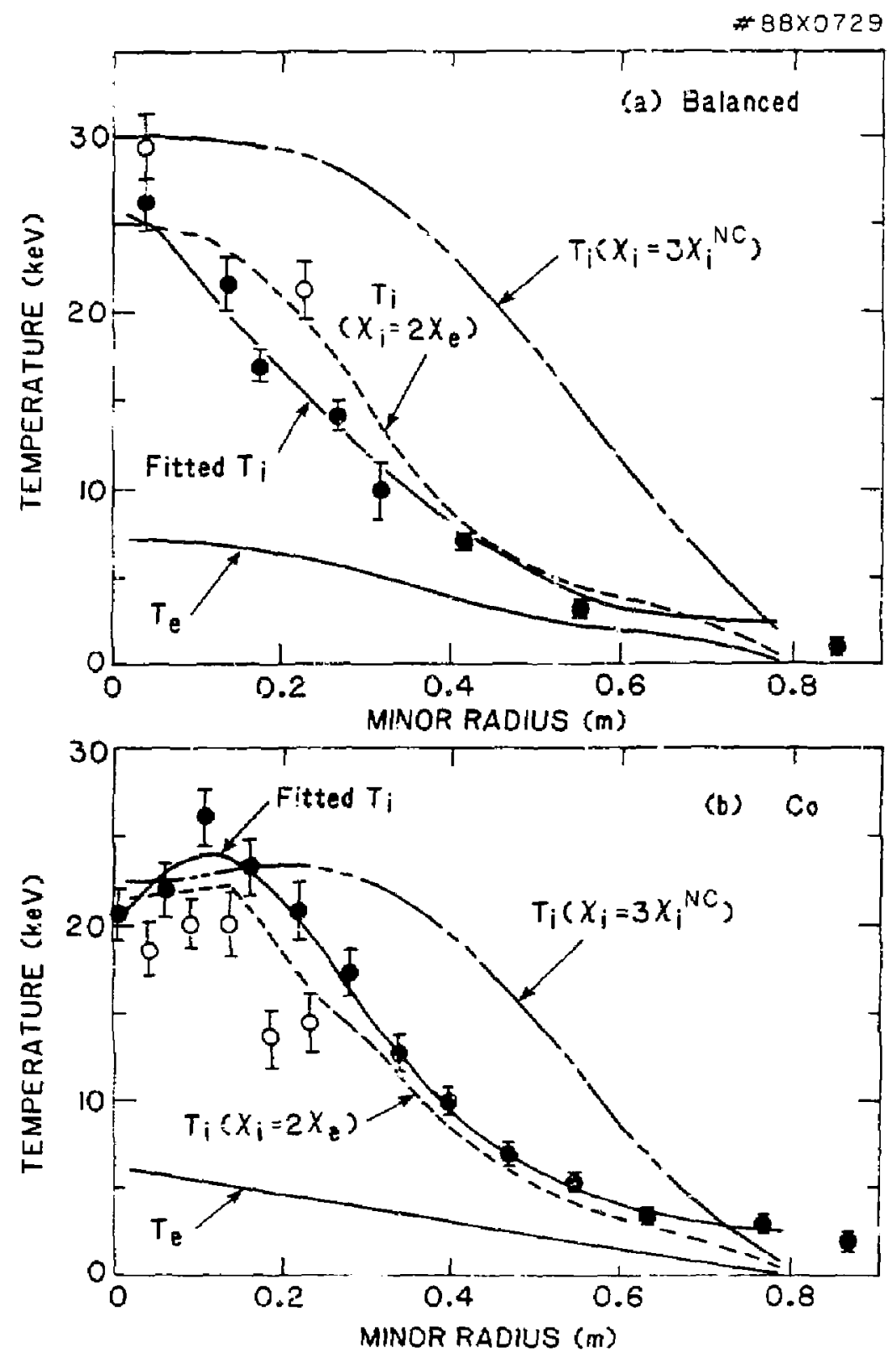

Fig. 1 

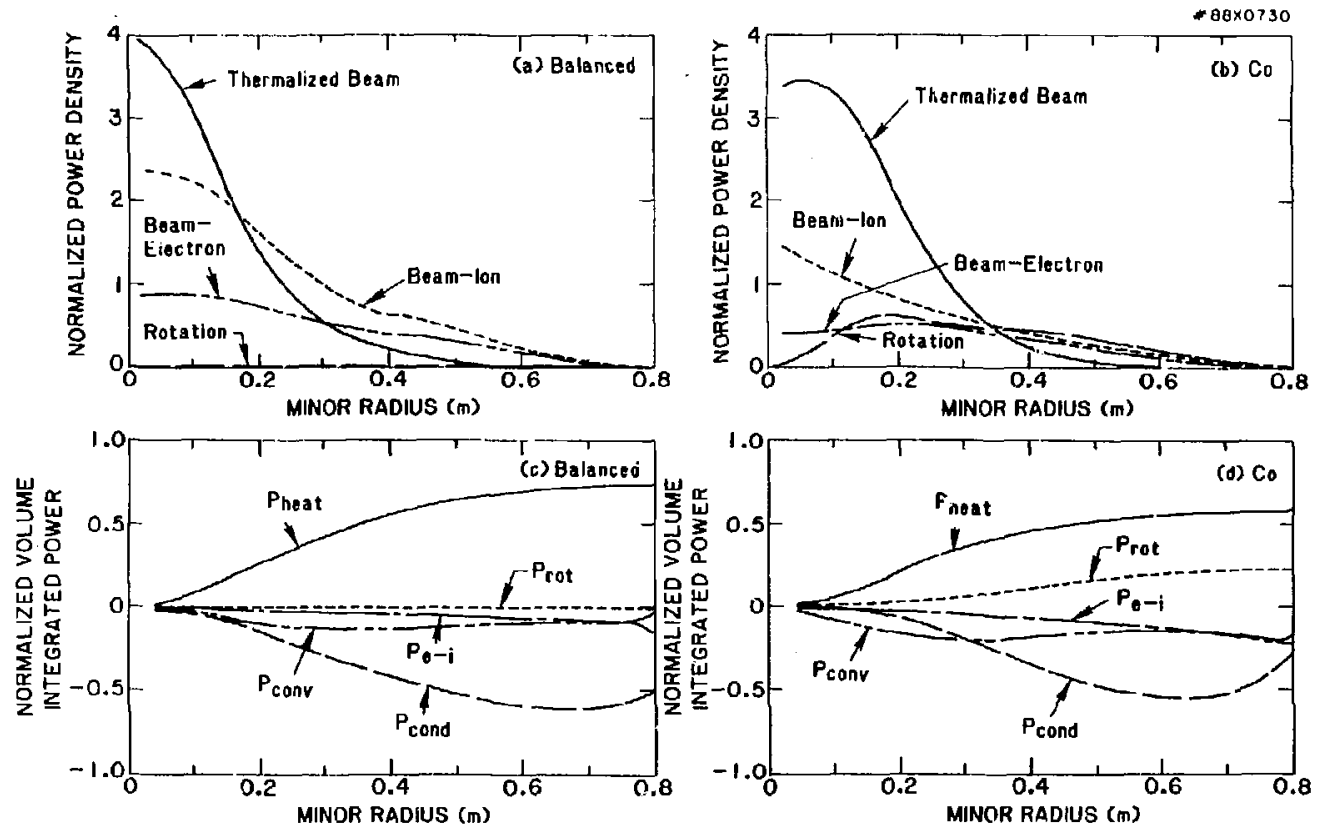

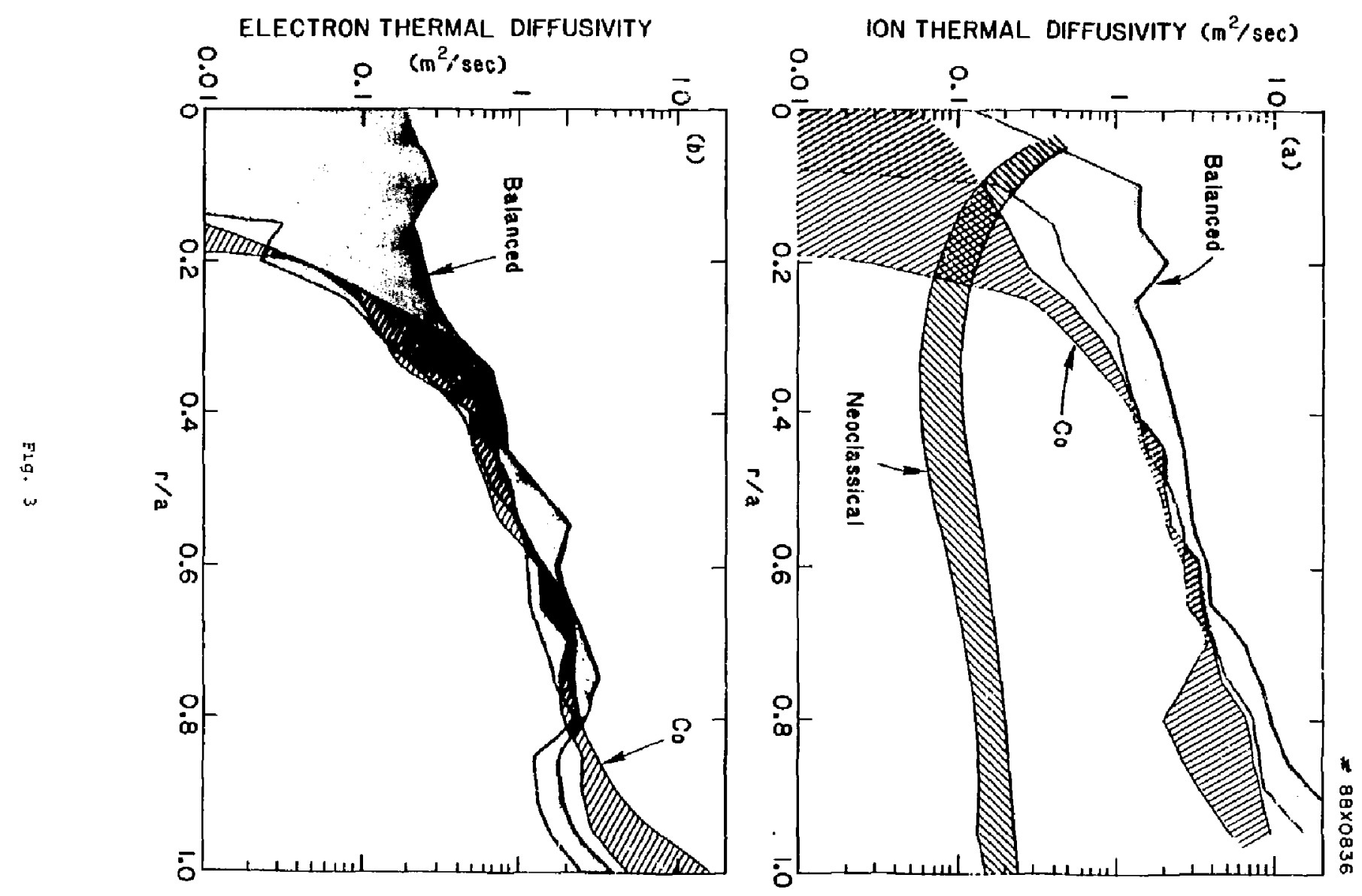